\title{
Relationship of serum vitamin D levels with coronary thrombus grade, TIMI flow, and myocardial blush grade in patients with acute ST-segment elevation myocardial infarction
}

\author{
Abdallah Ahmed Abdallah, Mohamed Ahmed Abd Elrhman, Ahmd Elshazly and Islam Bastawy* (D)
}

\begin{abstract}
Background: Vitamin D deficiency is a prevalent condition that is found in about $30-50 \%$ of the general population, and it is increasing as a new risk factor for coronary artery disease. Our study aimed to evaluate the relationship of serum vitamin D levels with coronary thrombus burden, Thrombolysis In Myocardial Infarction flow grade, and myocardial blush grade in patients managed by primary percutaneous coronary intervention for their first acute ST-segment elevation myocardial infarction.

Results: Eighty patients were included in the study with their first acute ST-segment elevation myocardial infarction and were managed by primary percutaneous coronary intervention. According to the serum concentrations of vitamin D, the study population was divided into 2 groups: group A with abnormal vitamin D levels less than 30 $\mathrm{ng} / \mathrm{ml}$ (50 patients) and group B with normal vitamin D levels equal to or more than $30 \mathrm{ng} / \mathrm{ml}$ (30 patients). Angiographic data was recorded before and after coronary intervention. On comparing thrombus grade and initial and post-procedural Thrombolysis In Myocardial Infarction flow between both groups of patients, there was no significant difference ( $p=0.327, p=0.692, p=0.397$ ). However, myocardial blush grade was better in patients with normal vitamin $\mathrm{D}$ levels $(p=0.029)$ without a significant correlation between vitamin $\mathrm{D}$ concentration values and myocardial blush grade $(r=0.164, p=0.146)$.

Conclusions: Patients with first acute ST-segment elevation myocardial infarction and normal vitamin D levels undergoing primary percutaneous coronary intervention had better myocardial blush grade and more successful microvascular reperfusion in comparison with patients with abnormal vitamin D levels. There was no significant difference between the normal and abnormal vitamin D groups regarding the coronary thrombus grade and Thrombolysis In Myocardial Infarction flow.
\end{abstract}

Keywords: Interventional cardiology, No-reflow, Vitamin D, Acute myocardial infarction

\footnotetext{
*Correspondence: islambastawy@hotmail.com

Department of Cardiology, Ain Shams University, Cairo, Egypt
}

\section{Springer Open}

(c) The Author(s). 2020 Open Access This article is licensed under a Creative Commons Attribution 4.0 International License, which permits use, sharing, adaptation, distribution and reproduction in any medium or format, as long as you give appropriate credit to the original author(s) and the source, provide a link to the Creative Commons licence, and indicate if changes were made. The images or other third party material in this article are included in the article's Creative Commons licence, unless indicated otherwise in a credit line to the material. If material is not included in the article's Creative Commons licence and your intended use is not permitted by statutory regulation or exceeds the permitted use, you will need to obtain permission directly from the copyright holder. To view a copy of this licence, visit http://creativecommons.org/licenses/by/4.0/. 


\section{Background}

Despite the medical and technological improvements in the re-vascularization procedures in coronary artery diseases (CAD), ST-segment elevation myocardial infarction (STEMI) remains a significant health concern. Primary percutaneous coronary intervention (PCI) is the favorable treatment option for restoring perfusion to the affected area of the myocardium as soon as possible. In STEMI, the incidence of no-reflow has been reported to be ranging between 11 and 41\%, depending on several clinical and angiographic factors in addition to the adopted definition of no-reflow [1]. The pathogenesis of no-reflow is known to be multi-factorial, and its possible mechanisms include injury related to ischemia, reperfusion, endothelial dysfunction, microvascular spasm, and distal thromboembolism [2].

Vitamin D deficiency causes endothelial dysfunction through its direct or indirect effect through the upregulation of the renin-angiotensin system or via induction of smooth muscle proliferation and a pro-inflammatory state [3]. The prevalence of vitamin D deficiency was estimated to be approximately $30-50 \%$ of the general population [4], and several studies have shown an association between vitamin $\mathrm{D}$ deficiency and cardiovascular diseases (CVD) including hypertension (HTN), acute myocardial infarction (AMI), heart failure, CAD, metabolic syndrome, and diabetes mellitus (DM) [5]. This study aimed to evaluate serum vitamin D level's relationship with coronary thrombus grade, Thrombolysis In Myocardial Infarction (TIMI) flow, and myocardial blush grade (MBG) in patients with first acute STEMI undergoing primary PCI.

\section{Methods}

This was a prospective cohort study that included 80 patients with their first acute STEMI who presented within $12 \mathrm{~h}$ after the onset of chest pain and referred for primary PCI in the period between December 2017 and November 2018.

Patients were excluded from the study if they had a previous history of ischemic heart disease (prior AMI, PCI, or coronary artery bypass graft), active infection, or malignancy. Patients on previous anti-thrombotic or anticoagulant medications and patients who received thrombolytic therapy before coronary angiography were also excluded from the study.

We applied the European Society of Cardiology guidelines for STEMI management in diagnosis [6]. A detailed history was taken, and a full clinical examination was done searching for CAD risk factors including age, sex, positive family history of premature CAD, body mass index, smoking, dyslipidemia, HTN, and DM.

Patients with DM were identified on admission as $\mathrm{HbA} 1 \mathrm{c} \geq 6.5 \%(48 \mathrm{mmol} / \mathrm{mol})$ or fasting plasma glucose $\geq 7.0 \mathrm{mmol} / \mathrm{l}(126 \mathrm{mg} / \mathrm{dl})$ or 2 -h postprandial glucose $\geq$ $11.1 \mathrm{mmol} / \mathrm{l}$ ( $\geq 200 \mathrm{mg} / \mathrm{dl}$ ) or those using either oral hypoglycemic agents or insulin treatment [7]. Dyslipidemia was defined as total cholesterol of at least $200 \mathrm{mg} / \mathrm{dl}$ or using anti-hyperlipidemic therapy on admission [8]. HTN was defined as office systolic blood pressure values $\geq 140 \mathrm{mmHg}$ and/or diastolic pressure values $\geq 90 \mathrm{mmHg}$ or using antihypertensive therapy on admission [9].

All patients received aspirin $300 \mathrm{mg}$ and clopidogrel $600 \mathrm{mg}$ (available P2Y12 inhibitor at our institute) before their transfer to the catheterization laboratory. Emergency coronary angiography was performed using the percutaneous femoral or radial approach. Heparin (100 $\mathrm{U} / \mathrm{kg}$ ) was administered in all patients during the primary PCI procedure [10].

The crossing of the occluded culprit artery was done using various guide wires, and pre-dilatation was performed via balloon angioplasty if necessary. Routine stenting using a drug-eluting stent was attempted directly or following balloon angioplasty. The usage of thrombus aspiration catheter and administration of tirofiban infusion were chosen according to the decision of the interventional cardiologist [11]. Thrombus grading was done using the TIMI thrombus scale grade [12].

The initial TIMI flow was assessed at the beginning of the procedure before wire crossing and the final TIMI flow immediately after the primary PCI using the TIMI flow grade classification [13]. The MBG was assessed visually on the angiogram and interpreted immediately after the primary coronary angioplasty procedure by the performing cardiologist: grade 0 , no myocardial blush; grade 1 , minimal myocardial blush; grade 2 , moderate myocardial blush; and grade 3, normal myocardial blush [14].

Ejection fraction (EF) was measured within the first $48 \mathrm{~h}$ after primary PCI by the modified Simpson's method using a (Vivid S5, General Electric Healthcare) echocardiography device.

We obtained peripheral venous samples on admission from all patients before the primary PCI then they were centrifuged early after collection, and extracted serum was stored at $-20{ }^{\circ} \mathrm{C}$. We measured 25 -hydroxy vitamin D concentrations (vitamin D) using the enzyme-linked immunosorbent assay (Calbiotech, 25(OH) Vitamin D ELISA).

According to serum vitamin D levels, study population was divided into 2 groups: group A, which included patients with abnormal vitamin D levels less than $30 \mathrm{ng} /$ $\mathrm{ml}$, and group $\mathrm{B}$, which included patients with normal vitamin D levels equal to or more than $30 \mathrm{ng} / \mathrm{ml}$.

\section{Statistical analysis}

We used version 20.0 of the Statistical Package for Social Sciences (SPSS Inc., Chicago, IL, USA) in the data analysis. Quantitative data was expressed as mean \pm standard deviation. Qualitative data was expressed as 
frequency and percentage. Comparing two means was done using the independent-samples $t$ test, the chisquare $\left(\chi^{2}\right)$ test was used in comparing proportions between qualitative parameters, and correlation between 2 sets of variables was done using the Spearman correlation coefficient $(r)$ test. The confidence interval was set to 95 , and the accepted margin of error was set to $5 \%$. So, the $p$ value was considered significant as follows: probability ( $p$ value) $\leq 0.05$ was considered significant, and $p$ value $>0.05$ was considered non-significant.

\section{Results}

Abnormal vitamin D levels $(<30 \mathrm{ng} / \mathrm{ml})$ were found in 50 patients representing group A while normal vitamin D levels $(\geq 30 \mathrm{ng} / \mathrm{ml})$ were found in 30 patients representing group $\mathrm{B}$.

\section{Baseline characteristics and clinical data}

There was no significant difference between both groups on comparing baseline characteristics and clinical data including age, gender, CAD risk factors, pain to door (PTD) time, door to balloon (DTB) time, and Killip class. Also, there was no significant difference between both groups on comparing EF measured within the first $48 \mathrm{~h}$ from primary PCI. This data was summarized in Table 1.

\section{Angiographic data}

A comparison between both groups regarding the initial angiography, final angiography, and interventional data was shown in Table 2. On comparing the number of affected vessels, culprit vessel, thrombus grade, initial TIMI flow, use of thrombus aspiration, or tirofiban infusion between both groups, we did not find any significant difference. Also, there was no significant difference in comparing post-procedural TIMI flow. However, there was a significant difference between both groups on comparing post-procedural MBG (Fig. 1), which was better within group B patients with normal vitamin D levels $(p=$ 0.02). Group A patients had MBG III in 20 patients (40\%), MBG II in 11 patients (22\%), MBG I in 13 patients (26\%), and MBG 0 in 6 patients (2\%) while group B patients had MBG III in 9 patients (30\%), MBG II in 16 patients (53.3\%), MBG I in 4 patients (26\%), and MBG 0 in 1 patient $(3.3 \%)(p=0.029)$. This significant difference was maintained when we compared the success of myocardial reperfusion between both groups. Patients with MBG II or III represented successful myocardial reperfusion, while patients with MBG 0 or I represented unsuccessful myocardial reperfusion. Successful myocardial reperfusion was achieved in 31patients of group A (62\%) versus 25 patients of group B (83.3\%) ( $p=0.047)$. However, on correlating serum vitamin $\mathrm{D}$ concentration values to $\mathrm{MBG}$, it was found to be non-significant $(r=0.164, p=0.146)$.

\section{Discussion}

Coronary revascularization is the main target of primary PCI for acute STEMI which is a life-threatening condition that requires fast diagnosis and proper intervention. However, a considerable number of patients still develop a no-reflow phenomenon during primary PCI having a

Table 1 Comparing baseline characteristics and clinical data between both groups

\begin{tabular}{|c|c|c|c|}
\hline Demographic data & Group A (vit. D < 30) $($ no. $=50)$ & Group B (vit. D > 30) $($ no. $=30)$ & $p$ value \\
\hline Age (years) & $54.82 \pm 7.55$ & $53.60 \pm 7.07$ & 0.476 \\
\hline \multicolumn{4}{|l|}{ Sex } \\
\hline Female & $13(26.0 \%)$ & $8(26.7 \%)$ & \multirow[t]{2}{*}{0.948} \\
\hline Male & $37(74.0 \%)$ & $22(73.3 \%)$ & \\
\hline Smoking & $18(36.00 \%)$ & $8(26.70 \%)$ & 0.388 \\
\hline HTN & $16(32.0 \%)$ & $10(33.3 \%)$ & 0.902 \\
\hline DM & $14(28.0 \%)$ & $8(26.7 \%)$ & 0.897 \\
\hline BMI $\left(\mathrm{kg} / \mathrm{m}^{2}\right)$ & $26.62 \pm 2.59$ & $26.70 \pm 2.93$ & 0.899 \\
\hline Dyslipidemia & $9(18.0 \%)$ & $5(16.7 \%)$ & 0.879 \\
\hline Positive family history & $8(16.0 \%)$ & $6(20.0 \%)$ & 0.649 \\
\hline PTD (h) & $7.500 \pm 2.39$ & $6.67 \pm 3.08$ & 0.210 \\
\hline DTB (min) & $73.00 \pm 9.15$ & $74.33 \pm 11.20$ & 0.564 \\
\hline $\mathrm{EF} \%$ & $42.26 \pm 7.48$ & $41.97 \pm 7.76$ & 0.867 \\
\hline \multicolumn{4}{|l|}{ Killip class } \\
\hline । & $44(88.0 \%)$ & 27 (90.0\%) & \multirow[t]{3}{*}{0.738} \\
\hline$\|$ & $5(10.0 \%)$ & $3(10.0 \%)$ & \\
\hline III & $1(2.0 \%)$ & $0(0.0 \%)$ & \\
\hline
\end{tabular}

HTN hypertension, DM diabetes mellitus, BMI body mass index, PTD pain to door, DTB door to balloon, EF ejection fraction 
Table 2 Comparing the initial angiography and interventional data between both groups

\begin{tabular}{|c|c|c|c|}
\hline Angiographic and interventional data & Group A (vit. D < 30) (no. = 50) & Group B (vit. D $\geq 30$ ) (no. = 30) & $p$ value \\
\hline \multicolumn{4}{|l|}{ Initial TIMI flow } \\
\hline 0 & $31(62.0 \%)$ & $15(50.0 \%)$ & \multirow[t]{4}{*}{0.692} \\
\hline । & $6(12.0 \%)$ & $4(13.3 \%)$ & \\
\hline$\|$ & $7(14.0 \%)$ & $7(23.3 \%)$ & \\
\hline III & $6(12.0 \%)$ & $4(13.3 \%)$ & \\
\hline \multicolumn{4}{|l|}{ Thrombus grading } \\
\hline 1 & $0(0.0 \%)$ & $2(6.7 \%)$ & \multirow[t]{5}{*}{0.327} \\
\hline 2 & $6(12.0 \%)$ & $5(16.7 \%)$ & \\
\hline 3 & $7(14.0 \%)$ & $3(10.0 \%)$ & \\
\hline 4 & $6(12.0 \%)$ & $5(16.7 \%)$ & \\
\hline 5 & $31(62.0 \%)$ & $15(50.0 \%)$ & \\
\hline \multicolumn{4}{|l|}{ Number of vessels } \\
\hline Single vessel & $39(78.0 \%)$ & $24(80.0 \%)$ & \multirow[t]{2}{*}{0.832} \\
\hline Multi-vessels & $11(22.0 \%)$ & $6(20.0 \%)$ & \\
\hline \multicolumn{4}{|l|}{ Culprit vessel } \\
\hline LAD & $31(62.0 \%)$ & $18(60.0 \%)$ & \multirow[t]{3}{*}{0.984} \\
\hline RCA & $11(22.0 \%)$ & $7(23.3 \%)$ & \\
\hline LCX & $8(16.0 \%)$ & $5(16.7 \%)$ & \\
\hline Thrombus aspiration & $3(6.0 \%)$ & $0(0.0 \%)$ & 0.171 \\
\hline Tirofiban infusion & 15 (30.0\%) & $5(16.7 \%)$ & 0.182 \\
\hline \multicolumn{4}{|l|}{ Post-PCI TIMI flow } \\
\hline । & $4(8.0 \%)$ & $1(3.3 \%)$ & \multirow[t]{3}{*}{0.397} \\
\hline$\|$ & $11(22.0 \%)$ & $4(13.3 \%)$ & \\
\hline III & $35(70.0 \%)$ & 25 (83.3\%) & \\
\hline \multicolumn{4}{|l|}{ MBG } \\
\hline 0 & $6(12.0 \%)$ & $1(3.3 \%)$ & \multirow[t]{4}{*}{$0.029^{*}$} \\
\hline । & $13(26.0 \%)$ & $4(13.3 \%)$ & \\
\hline$\|$ & $11(22.0 \%)$ & $16(53.3 \%)$ & \\
\hline III & $20(40.0 \%)$ & $9(30.0 \%)$ & \\
\hline \multicolumn{4}{|l|}{ Myocardial reperfusion } \\
\hline Unsuccessful (MBG 0, I) & 19 (38.0\%) & $5(16.7 \%)$ & \multirow[t]{2}{*}{$0.047^{*}$} \\
\hline Successful (MBG II, III) & $31(62.0 \%)$ & 25 (83.3\%) & \\
\hline
\end{tabular}

* = significant

TIMThrombolysis In Myocardial Infarction, MBG myocardial blush grade

worse outcome [15]. Several studies showed that vitamin D deficiency was prevalent in AMI patients, and they indicated worse STEMI outcomes in those patients with vitamin D deficiency $[16,17]$.

\section{Abnormal vitamin D levels and myocardial infarction}

Our study showed that patients with acute STEMI had a higher prevalence of abnormal vitamin D levels in comparison with the general population as abnormal vitamin $\mathrm{D}$ levels (insufficient or deficient) were found in $62.5 \%$ of the study population. This observation is supported by the study of Lee and his colleagues in 2011 that showed even higher rates of abnormal vitamin D levels in AMI patients as 96\% had abnormal vitamin D levels [18]. Also, Mahdavi and his colleagues in 2013 have observed vitamin D deficiency in most of the patients with acute coronary syndrome, and normal vitamin D levels were found only in $10.6 \%$ of patients [19]. Similar results were presented by Correia and his colleagues in 2013 in their study that mentioned that vitamin D levels were normal only in $10 \%$ of patients with AMI [20].

\section{Abnormal vitamin $D$ levels and angiographic findings} We did not find in our study a significant difference between both groups regarding the number of affected 


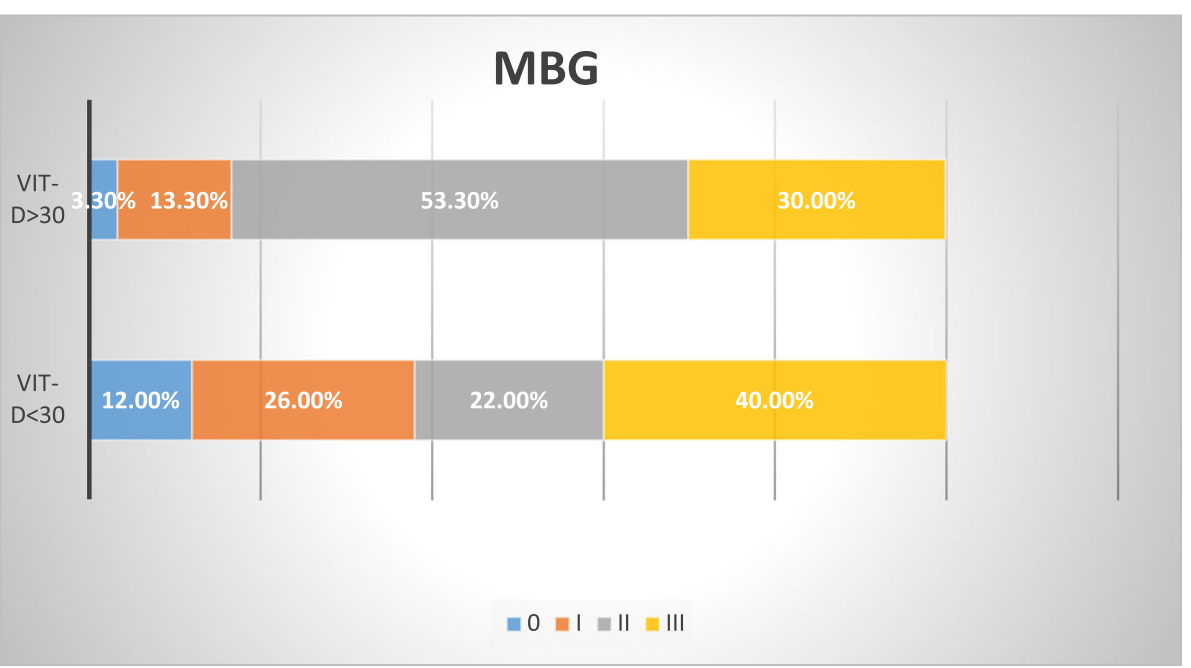

Fig. 1 Comparing MBG between both groups

vessels which is matching with the study conducted by Goleniewska and his colleagues in 2013 that did not find a significant difference in vitamin D levels among STEMI patients with single or multi-vessel affection [21].

It is important to mention that combining postprocedural TIMI flow and MBG is more accurate in the angiographic assessment of no-reflow as post-procedural TIMI flow III reflects successful epicardial reperfusion that is not always associated with successful microvascular or myocardial tissue reperfusion that is achieved by having MBG II or III [22, 23]. Despite the restoration of epicardial flow in a considerable number of patients (TIMI III flow), impaired myocardial reperfusion (MBG 0 or I) is associated with poor outcomes [24].

Our study did not find a significant difference in comparing coronary thrombus grade and initial or postprocedural TIMI flow between both groups. However, MBG was significantly higher in patients with normal vitamin D levels, but there was no significant linear correlation between vitamin D levels and MBG. Cerit and his colleagues in 2019 conducted a study that showed that the mean values of vitamin D levels were lower in patients who developed no-reflow; however, vitamin D values were not shown to be an independent predictor of no-reflow that was defined by achieving TIMI flow less than III [25]. This may point to a potential role of abnormal vitamin D levels in the reduction of successful reperfusion at the microvascular level rather than affecting epicardial coronary thrombus grade or epicardial reperfusion. What supports our hypothesis is that high residual platelet reactivity measured during primary PCI is associated with more incidence of no-reflow [26], and patients with vitamin D deficiency in the study of Verdoia and her colleagues in 2016 had higher residual platelet reactivity with adenosine diphosphate (ADP) when measured 30-90 days after coronary intervention in patients kept on dual antiplatelets raising concerns that vitamin D deficiency may impair ADP antagonists' effectiveness and it may have a potential role in increasing risk of clopidogrel resistance [27].

On the other hand, our explanation of the absence of a significant linear correlation between vitamin D concentration values and MBG is that the clinical importance of MBG relies on being either MBG 0 or I versus MBG II or III rather than on the exact MBG. And, in our study, group A with abnormal vitamin D levels had a higher percentage of MBG III. However, on comparing the overall success of microvascular reperfusion, it was significantly higher in group B with normal vitamin D levels.

Moreover, a study conducted by Şen and his colleagues in 2017 that evaluated the association between vitamin D levels and grade of collateralization in STEMI patients found that vitamin D levels were higher among patients with well-developed collaterals that are dependent on various endogenous mediators such as vascular endothelial growth factor, nitric oxide, and other neuro-humoral markers [28].

\section{Abnormal vitamin D levels and left ventricular ejection fraction}

Despite achieving better myocardial reperfusion in patients with normal vitamin D levels, we did not find a significant difference between both groups in EF measured within the first $48 \mathrm{~h}$ after primary PCI, which is consistent with the findings of a study conducted by Khalili and his colleagues in 2012 that did not find an association between LV systolic function and vitamin D levels [29]. This may be due to early assessment of LV 
functions after AMI within the first $48 \mathrm{~h}$ where myocardial stunning is found. However, later assessment of LV function may show a difference as shown in the study of Rehman and his colleagues in 2020 that showed significantly higher LV EF in STEMI patients with higher MBG when LV function was assessed 3 months after the primary PCI [30].

\section{Study limitations}

This was a single-center study, and further studies with a larger number of patients are needed to confirm our findings and to assess minor differences seen in thrombus grading between both groups. Also, our population included only one geographical region. It is also important to mention that vitamin D status can change with various factors, such as seasonal variation, geography, latitude, and sunlight exposure.

\section{Conclusion}

Patients with first acute STEMI and normal vitamin D levels undergoing primary PCI had better MBG and more successful microvascular reperfusion in comparison with patients with abnormal vitamin D levels. There was no significant difference between normal and abnormal vitamin $\mathrm{D}$ groups regarding the coronary thrombus grade and TIMI flow.

\section{Abbreviations}

ADP: Adenosine diphosphate; AMI: Acute myocardial infarction; BMI: Body mass index; CAD: Coronary artery disease; CVD: Cardiovascular diseases; DM: Diabetes mellitus; DTB: Door to balloon; EF: Ejection fraction; HTN: Hypertension; LAD: Left anterior descending artery; LCX: Left circumflex artery; LV: Left ventricular; MBG: Myocardial blush grade; PCI: Percutaneous coronary intervention; PTD: Pain to door; RCA: Right coronary artery; STEMI: ST-segment elevation myocardial infarction; TIMI: Thrombolysis In Myocardial Infarction

\section{Acknowledgements}

Not applicable.

\section{Authors' contributions}

AAA was a major contributor to the data collection in addition to the writing of the manuscript. MAA revised the dataset and participated in the data analysis. AE was the main contributor to the study design in addition to participation in the data analysis. IB was the main contributor to the revision and analysis of the patient data, statistical analysis, and writing of the manuscript. All authors read and approved the final manuscript.

\section{Funding}

No funds were received to fulfill this work.

\section{Availability of data and materials}

The datasets used and analyzed during the current study are available from the corresponding author on reasonable request.

\section{Ethics approval and consent to participate}

Approval of the Ain Shams University ethical committee was obtained for this study (Committee reference number is not available); written informed consent was provided by all patients participating in the study.

\section{Consent for publication}

Not applicable.

\section{Competing interests}

The authors declare that they have no competing interests.

Received: 8 September 2020 Accepted: 10 November 2020

Published online: 23 November 2020

\section{References}

1. Harrison RW, Aggarwal A, Ou F et al (2013) Incidence and outcomes of noreflow phenomenon during percutaneous coronary intervention among patients with acute myocardial infarction. Am J Cardiol 111(2):178-184

2. Bouleti C, Mewton N, Germain S et al (2015) The no-reflow phenomenon: state of the art. Arch Cardiovasc Dis 108(12):661-674

3. Jaffe R, Charron T, Puley G et al (2008) Microvascular obstruction and the no-reflow phenomenon after percutaneous coronary intervention. Circulation 117(24):3152-3156

4. Lee $J H$, O'Keefe $J H$, Bell D et al (2008) Vitamin D deficiency: an important, common, and easily treatable cardiovascular risk factor. J Am Coll Cardiol 52(24):1949-1956

5. Kheiri B, Abdalla A, Osman M et al (2018) Vitamin D deficiency and risk of cardiovascular diseases: a narrative review. Clin Hypertens 24(1):1-9

6. Bax JJ, Baumgartner $\mathrm{H}$, Ceconi $\mathrm{C}$ et al (2012) Third universal definition of myocardial infarction. J Am Coll Cardiol 60(16):1581-1598

7. American Diabetes Association (2019) 2. Classification and diagnosis of diabetes: standards of medical care in diabetes-2019. Diabetes Care 42: S13-S28

8. Ference B, Graham I, Tokgozoglu L et al (2018) Reprint of: Impact of lipids on cardiovascular health: JACC Health Promotion Series. J Am Coll Cardiol 72(23 Part B):2980-2995

9. Lurbe E, Agabiti-Rosei E, Cruickshank JK et al (2016) (2016) European Society of Hypertension guidelines for the management of high blood pressure in children and adolescents. J Hypertens 34:1887-1920

10. Schulz S, Richardt G, Laugwitz KL et al (2014) Prasugrel plus bivalirudin vs. clopidogrel plus heparin in patients with ST-segment elevation myocardial infarction. Eur Heart J 35(34):2285-2294

11. Gaspar A, Lourenço AP, Pereira MÁ et al (2018) Randomized controlled trial of remote ischaemic conditioning in ST-elevation myocardial infarction as adjuvant to primary angioplasty (RIC-STEMI). Basic Res Cardiol 113(3):14

12. Gibson CM, de Lemos JA, Murphy SA et al (2001) Combination therapy with abciximab reduces angiographically evident thrombus in acute myocardial infarction: a TIMI 14 substudy. Circulation 103(21):2550-2554

13. Chesebro JH, Knatterud G, Roberts R et al (1987) Thrombolysis in Myocardial Infarction (TIMI) Trial, phase l: a comparison between intravenous tissue plasminogen activator and intravenous streptokinase. Clinical findings through hospital discharge. Circulation 76(1):142-154

14. Henriques JP, Zijlstra F, Hof AW et al (2003) Angiographic assessment of reperfusion in acute myocardial infarction by myocardial blush grade. Circulation 107(16):2115-2119

15. Rezkalla SH, Kloner RA (2008) Coronary no-reflow phenomenon: from the experimental laboratory to the cardiac catheterization laboratory. Catheter Cardiovasc Interv 72(7):950-957

16. Machulsky NF, Barchuk M, Gagliardi J et al (2018) Vitamin D is related to markers of vulnerable plaque in acute myocardial infarction. Curr Vasc Pharmacol 16(4):355-360

17. Milazzo V, De Metrio M, Cosentino N et al (2017) Vitamin D and acute myocardial infarction. World J Cardiol 9(1):14-20

18. Lee JH, Gadi R, Spertus JA et al (2011) Prevalence of vitamin D deficiency in patients with acute myocardial infarction. Am J Cardiol 107(11):1636-1638

19. Mahdavi K, Amirajam Z, Yazdankha S et al (2013) The prevalence and prognostic role of vitamin $D$ deficiency in patients with acute coronary syndrome: a single centre study in south-west of Iran. Heart Lung Circ 22(5): 346-351

20. Correia LC, Sodré F, Garcia G et al (2013) Relation of severe deficiency of vitamin $\mathrm{D}$ to cardiovascular mortality during acute coronary syndromes. Am J Cardiol 111(3):324-327

21. Goleniewska B, Kacprzak M, Zielińska M (2014) Vitamin D level and extent of coronary stenotic lesions in patients with first acute myocardial infarction. Cardiol J 21(1):18-23

22. Gupta S, Gupta MM (2016) No-reflow phenomenon in percutaneous coronary interventions in ST-segment elevation myocardial infarction. Indian Heart J 68(4):539-551 
23. Ramjane K, Han L, Jin C (2008) The diagnosis and treatment of the noreflow phenomenon in patients with myocardial infarction undergoing percutaneous coronary intervention. Exp Clin Cardiol 13(3):121

24. Kampinga MA, Nijsten MW, Gu YL et al (2010) Is the myocardial blush grade scored by the operator during primary percutaneous coronary intervention of prognostic value in patients with ST-elevation myocardial infarction in routine clinical practice? Circ Cardiovasc Interv 3(3):216-223

25. Cerit L, Kemal H, Cerit Z et al (2019) Is there any link between vitamin D and coronary no-reflow phenomenon? Prog Nutr 21(2):270-275

26. Aitmokhtar O, Paganelli F, Benamara S et al (2017) Impact of platelet inhibition level on subsequent no-reflow in patients undergoing primary percutaneous coronary intervention for ST-segment elevation myocardial infarction. Arch Cardiovasc Dis 110(11):626-633

27. Verdoia M, Pergolini P, Rolla R et al (2016) Vitamin D levels and highresidual platelet reactivity in patients receiving dual antiplatelet therapy with clopidogrel or ticagrelor. Platelets 27(6):576-582

28. Sen Ö, Topuz M, Acele A et al (2017) The influence of plasma 25-(OH) vitamin D levels in acute ST elevation myocardial infarction. Cardiol J 24(6): 677-684

29. Khalili H, Talasaz AH, Salarifar M (2012) Serum vitamin D concentration status and its correlation with early biomarkers of remodeling following acute myocardial infarction. Clin Res Cardiol 101(5):321-327

30. Rehman AU, Malik J, Javed N et al (2020) Myocardial blush grade: a determinant of left ventricular ejection fraction and adverse outcomes in STEMI. Scott Med J. https://doi.org/10.1177/0036933020941260

\section{Publisher's Note}

Springer Nature remains neutral with regard to jurisdictional claims in published maps and institutional affiliations.

\section{Submit your manuscript to a SpringerOpen ${ }^{\circ}$ journal and benefit from:}

- Convenient online submission

- Rigorous peer review

- Open access: articles freely available online

- High visibility within the field

- Retaining the copyright to your article

Submit your next manuscript at $\boldsymbol{\nabla}$ springeropen.com 